Service social

\title{
Intervention de groupe auprès de la population itinérante de Montréal
}

\section{François Thivierge}

Volume 43, numéro 2, 1994

Enfants, parents, intervenants

URI : https://id.erudit.org/iderudit/706661ar

DOI : https://doi.org/10.7202/706661ar

Aller au sommaire du numéro

Éditeur(s)

École de service social de l'Université Laval

ISSN

1708-1734 (numérique)

Découvrir la revue

Citer cet article

Thivierge, F. (1994). Intervention de groupe auprès de la population itinérante de Montréal. Service social, 43(2), 147-157. https://doi.org/10.7202/706661ar d'utilisation que vous pouvez consulter en ligne.

https://apropos.erudit.org/fr/usagers/politique-dutilisation/ 


\section{CONTRIBUTION SPÉCIALE}

Le Fonds Simone-Paré a été créé en 1990 en l'honneur de madame Simone Paré, professeure à l'École de service social de l'Université Laval de 1947 à 1983. Il vise à promouvoir le développement du service social des groupes en appuyant financièrement des activités d'enseignement et de recherche; en outre, le Fonds a créé un prix annuel visant à souligner une contribution particulière au développement de l'intervention en service social des groupes. Le prix a été attribué pour la première fois au printemps 1994; monsieur François Thivierge du Centre d'accueil Préfontaine de Montréal en a été le récipiendaire. Dans le texte qui suit, il précise l'orientation de ses interventions auprès de la population itinérante de Montréal.

\section{Intervention de groupe auprès de la population itinérante de Montréal}

FRANÇOIS THIVIERGE Centre d'accueil Préfontaine

\section{INTRODUCTION}

Afin de mieux situer l'intervention du service communautaire du Centre d'accueil Préfontaine auprès de la population itinérante, je vous présente deux projets d'intervention importants: le travail de rue et la création d'un groupe d'entraide, le groupe communautaire l'Itinéraire. 


\section{LE TRAVAIL DE RUE (1986-1989)}

En 1986, lorsque nous avons mis sur pied le service communautaire du Centre d'accueil Préfontaine, un des objectifs était de rejoindre dans leur milieu de vie les personnes itinérantes alcooliques et toxicomanes qui formaient le «noyau dur» de l'itinérance. La plupart d'entre elles vivaient dans la rue au centre-ville de Montréal, alors que d'autres avaient une chambre dans des immeubles délabrés du secteur. Le travail de rue consistait à rencontrer les personnes itinérantes dans la rue, dans certains secteurs bien délimités du centre-ville, sur les bouches d'aération d'édifices, dans des lieux publics ainsi que dans leur chambre lorsqu'elles en avaient une. Ce contact direct dans le milieu nous a permis d'évaluer les besoins des personnes itinérantes et d'élaborer par la suite des pistes de solution à leurs divers problèmes. De 1986 à 1989, nous avons assuré un suivi auprès d'une centaine de personnes itinérantes.

Ce suivi consistait à jouer un rôle d'advocacy (promotion et défense des droits) en faveur de ces personnes qui subissaient des abus et des rejets de toutes sortes. Dans le cadre de ce travail, nous avons eu à faire des démarches pour obtenir des prestations d'aide sociale pour les personnes itinérantes sans adresse; à témoigner à des procès pour obtenir des peines moins sévères et des travaux compensatoires; à dénoncer les listes noires officielles et officieuses qui empêchaient certaines personnes itinérantes d'obtenir des services de santé et des services sociaux adéquats et à défendre auprès de plusieurs instances le droit des personnes itinérantes à avoir accès à un logement salubre et sécuritaire à un coût raisonnable.

\subsection{De l'intervention individuelle vers une démarche d'empowerment}

Parallèlement à l'intervention individuelle utilisée dans le travail de rue, nous avons fait appel à l'intervention de groupe. À travers une démarche d'empowerment (accès au pouvoir), notre objectif était alors de sensibiliser les personnes itinérantes à leurs droits et de les encourager à promouvoir et à défendre ces droits. Pour atteindre ce but, nous avons sensibilisé et organisé des groupes de personnes itinérantes pour qu'elles participent à diverses manifestations, chacune des activités visant un objectif bien particulier, à savoir :

1. La participation d'un groupe de personnes itinérantes au comité consultatif sur les sans-abri de la Ville de Montréal (1987) leur a permis d'être reconnues comme citoyens et citoyennes et $d^{\prime} y$ revendiquer leurs droits. 
2. La participation d'un groupe de personnes itinérantes à la journée d'échanges du Réseau d'aide aux personnes seules et itinérantes de Montréal (1987) leur a permis d'exprimer des opinions sur leurs problèmes et d'élaborer diverses solutions.

3. Pour faire respecter leurs droits de locataire alors qu'elles étaient menacées d'éviction par un propriétaire de maisons de chambres du centre-ville, des personnes itinérantes ont participé à la mise sur pied d'un comité de locataires (1987).

4. Des personnes itinérantes ont exprimé leurs opinions sur l'année internationale des sans-abri (1987) et sensibilisé la population à leur vécu en participant à deux émissions de télévision animées par Jeannette Bertrand, Parler pour parler, diffusées sur les ondes de Radio-Québec (1988).

Le fait d'axer le travail de ces personnes sur leurs compétences plutôt que sur leurs problèmes a eu un impact sur le choix d'activités basées sur l'action et a été un élément qui, à notre avis, a fait que le groupe a pu être rejoint.

\section{LA FORMATION DU GROUPE L'ITINÉRAIRE: LA RÉALISATION D'UNE PRATIQUE D'EMPOWERMENT (1989 À AUJOURD'HUI)}

Une démarche visant à redonner le pouvoir aux personnes ne s'improvise pas et ne naît pas du jour au lendemain. Notre expérience a débuté par l'intervention individuelle pour déboucher assez tôt sur l'intervention de groupe avec une approche d'empowerment. Par la suite, afin de répondre adéquatement aux besoins identifiés par les personnes itinérantes, nous avons participé comme membres de trois organismes sans but lucratif d'habitation à l'achat et à la rénovation de quatre maisons de chambres au centre-ville de Montréal. Cette implication a permis d'apporter une réponse aux besoins de base des personnes itinérantes, notamment par l'obtention de prestations d'aide sociale et par l'accès à une chambre ou à un studio rénovés pour certaines personnes. Le fait de répondre à des besoins concrets a contribué à la stabilisation d'une clientèle reconnue dans le milieu comme faisant partie du noyau dur de l'itinérance.

\subsection{Mise sur pied d'un groupe d'entraide}

En 1988, quelques personnes itinérantes ont commencé à nous faire part de leur désir d'avoir accès à un local ouvert où les membres 
pourraient s'entraider à l'occasion de rencontres et à travers leur participation à des activités. En 1989, nous avons effectué des démarches auprès de différents organismes, afin de mettre nos efforts en commun et ce, dans le but d'ouvrir un groupe d'entraide pour les personnes itinérantes. Ces premières tentatives n'eurent pas de suite. Les organismes croyaient que leurs philosophies d'intervention ne seraient pas respectées dans la mise sur pied d'un tel projet. En 1990, une consultation auprès des personnes itinérantes nous a amenés à élaborer un projet de groupe d'entraide qui fut présenté au conseil d'administration du Centre d'accueil Préfontaine, dans le but d'y faire voter les budgets nécessaires à son fonctionnement durant ses trois premières années d'existence. Le projet fut entériné par le conseil d'administration et l'ouverture du groupe d'entraide eut lieu en août 1990 dans le quartier Centre-Sud de Montréal. Les objectifs visés par ce projet étaient:

1. de combattre l'isolement que vivent ces personnes dans leur chambre;

2. d'éviter les rechutes;

3. de permettre aux personnes de s'aider les unes les autres, en échangeant des idées et en partageant des sentiments et des informations ainsi que leurs expériences;

4. d'augmenter le niveau de socialisation des personnes à travers les échanges et les activités organisées au local ;

5. d'accroître le sens des responsabilités par une prise en charge de l'organisation du local ;

6. de susciter un sentiment d'appartenance ;

7. de permettre à ces personnes d'avoir accès à un lieu de prise d'initiative et de créativité ;

8. de développer la conscientisation sociale.

Ces objectifs ont servi de guide pour élaborer la programmation du projet qui a été axée autour de quatre fonctions :

1. Fonction récréative

Activités: jeux de société, divers ateliers (ex.: réparation de vélos), sorties de groupe (La Ronde, cinéma, etc.) et autres activités sportives et culturelles.

2. Fonction d'épanouissement personnel et d'entraide Activités: café-rencontre, cuisine collective, rencontres d'échange et d'information sur divers sujets et activités connexes. 


\section{Conscientisation sociale}

Activités : rencontres d'échange et d'information sur divers sujets d'ordre collectif, participation à des actions collectives pour dénoncer et tenter de résoudre certaines situations problématiques liées aux personnes itinérantes.

4. Fonction de promotion et création d'organismes connexes Activités : mise sur pied d'organismes qui permettront aux personnes itinérantes de reprendre le pouvoir sur leur vie et de se doter d'outils qui faciliteront leur réinsertion sociale.

\subsection{Actualisation du projet de groupe d'entraide}

Les membres du groupe sont des personnes qui vivent divers problèmes (alcoolisme et toxicomanie, problèmes de santé physique et mentale, désengagement social, problèmes judiciaires et de logement). D'ailleurs, une des plus grandes difficultés dans la mise en œuvre du projet fut I'instabilité du membership du groupe en raison des nombreuses rechutes dues aux problèmes d'alcoolisme et de toxicomanie. Ainsi, il a fallu un an avant que le conseil d'administration soit opérationnel et que les postes soient occupés en majorité par des personnes itinérantes; il en fut de même pour la réalisation de tous les autres projets et activités du groupe. Cependant, après quatre années d'existence, le groupe d'entraide demeure un groupe ouvert, mais avec un noyau de base stabilisé.

Une autre difficulté importante fut de réussir à concilier les objectifs d'intervention visant le développement d'une conscientisation sociale et le besoin légitime des membres du groupe de voir rapidement des résultats concrets de leur action. Par exemple, dans le cadre de notre intervention, nous devions nous fixer des objectifs à moyen et à long terme, car des résultats à court terme avec une population si démunie n'étaient pas envisageables. Par contre, pour répondre aux attentes des membres du groupe, nous devions favoriser l'obtention d'une réponse à leurs besoins à court terme, afin qu'elles vivent une valorisation immédiate et qu'elles puissent poursuivre leur démarche. Deux projets furent donc mis sur pied: le souper collectif du dimanche et l'ouverture du café-rencontre. Ces projets permirent aux membres d'obtenir dans l'immédiat des résultats concrets de leur participation au groupe d'entraide et servirent de catalyseurs auprès des membres du groupe. La revalorisation acquise dans la réalisation de ces premiers projets a motivé certains membres à accepter de prendre part à l'élaboration de projets dont les résultats n'apparaîtront qu'à moyen et à long terme. 
Aux projets formels s'est ajoutée une activité informelle, c'està-dire qu'elle s'imposa d'elle-même, hors des structures mises en avant par le groupe. C'est aux réunions du groupe d'entraide, animées par les intervenants et les personnes itinérantes, que se rassemblaient les membres du groupe. Ces réunions avaient lieu, au besoin, quand la pression devenait trop forte au sein du groupe, qu'il devenait urgent de régler certains problèmes et de consulter les membres sur certaines décisions prises ou à prendre. Ce forum fut un lieu qui favorisa la participation de tous les membres à l'élaboration des projets du groupe. Ce fut également le premier lieu véritable de partage du pouvoir entre les intervenants et les personnes itinérantes. L'exercice de la démocratie à travers cette activité informelle a permis aux personnes itinérantes de faire l'apprentissage du pouvoir et de l'exercer par la suite à l'intérieur des structures de l'organisme.

$C^{\prime}$ est par leur implication dans la réalisation de ces activités et de ces projets que les membres ont acquis à la fois une autonomie et une identité collective. C'est par l'apprentissage du pouvoir qu'ils ont développé leurs capacités et acquis les moyens nécessaires à leur propre épanouissement et à celui du groupe d'entraide. Notre intervention auprès des membres du groupe a toujours été faite dans la perspective de leur laisser prendre le pouvoir; nous avons incité les personnes à prendre des initiatives et à développer leur créativité dans le but de susciter une autovalorisation. Vouloir favoriser l'accès au pouvoir passe par des gestes très concrets qui ont pourtant une symbolique très profonde; dans le cas du groupe d'entraide, le fait de remettre les clefs du local aux membres a été un point tournant. Dans une démarche d'empowerment auprès des personnes itinérantes, on peut penser que ce geste a probablement permis la continuité du projet et a préparé les personnes à s'autogérer et à accéder au pouvoir.

\subsection{Activités et projets du groupe d'entraide}

L'élaboration du groupe s'est faite par la mise sur pied par les membres de divers projets et activités. En plus de viser la réalisation des objectifs du projet du groupe d'entraide, chaque activité et chaque projet comportaient également des objectifs particuliers. L'atteinte de tous ces objectifs s'est faite graduellement au fur et à mesure de la réalisation des activités et des projets suivants: 
1. Conseil d'administration (1990)

Objectif particulier: permettre aux membres de faire l'apprentissage du pouvoir.

Activités: le conseil d'administration, qui compte sept membres dont cinq sont des personnes itinérantes, voit à la gestion de l'organisme et à la coordination de I'ensemble des activités de l'organisme. Les membres sont élus à l'assemblée générale qui se tient une fois par année.

Les membres du conseil d'administration représentent l'organisme auprès de diverses instances, pour y faire la promotion des activités du groupe.

Fréquence des activités: une réunion du conseil d'administration au moins une fois par mois. Les membres assurent la gestion de l'organisme au quotidien (administration, coordination, représentation, trésorerie et secrétariat).

Nombre de participants: depuis 1990, environ 25 personnes.

\section{Souper collectif (1990 à 1991)}

Objectif particulier : permettre aux membres de manger et d'apprendre à cuisiner un repas complet et équilibré.

Activités: cotisation des membres pour l'achat de la nourriture. Chaque semaine, préparation d'un repas communautaire au local par différentes équipes.

Fréquence des activités: une fois par semaine.

Nombre de participants: pour la durée du projet, environ 100 personnes.

\section{Café-rencontre (depuis 1990)}

Objectif particulier: permettre aux membres d'avoir accès à un lieu de rencontres, d'échanges et d'entraide.

Activités: le local est ouvert sur une base bénévole par un membre qui y accueille les visiteurs et les informe sur les diverses activités du groupe. Café vendu sur place à $0,10 \$$ la tasse. Utilisation gratuite du téléphone.

Fréquence des activités : cinq jours par semaine, de $9 \mathrm{~h}$ à $16 \mathrm{~h}$.

Nombre de participants: depuis 1990, environ 300 personnes. 
4. Réunions du groupe d'entraide (1991 à 1993)

Objectif particulier : cette activité $n^{\prime}$ ayant pas été planifiée, il n'y a donc pas eu d'objectif particulier établi au préalable. A posteriori, cette activité favorisa la prise en charge du groupe par les personnes itinérantes et elle fut un lieu privilégié d'apprentissage du pouvoir.

Activités: réunions des membres de l'organisme qui sont animées conjointement par les intervenants et les personnes itinérantes. Les problèmes vécus à l'intérieur des activités et des projets sont abordés; des pistes de solution sont proposées par les membres. De nouveaux projets et de nouvelles activités sont également élaborés; les décisions prises sont acheminées par la suite au conseil d'administration qui doit les entériner avant qu'elles n'entrent en vigueur.

Fréquence des activités: au besoin, environ dix réunions en 1991, six en 1992 et quatre en 1993.

Nombre des participants: pour la durée du projet, environ 200 personnes.

5. Comité des locataires (depuis 1992)

Objectif particulier: permettre aux membres d'acquérir les moyens et les instruments nécessaires à la promotion et à la défense de leurs droits de locataire.

Activités: le comité des locataires a été à la base de I'organisation des locataires de l'OSBL Villa Exprès pour Toi, qui subissaient des abus de pouvoir et qui ont revendiqué et défendu par la suite leurs droits auprès de la Régie du logement, de la Société d'habitation du Québec et de la Régie régionale de Montréal-Centre.

Fréquence des activités: une réunion du comité exécutif au moins une fois par mois. Une réunion de tous les locataires au moins quatre fois par année.

Nombre de participants: depuis 1992, environ 150 personnes. 


\section{Journal L'Itinéraire (depuis 1992)}

Objectifs particuliers : développer un outil de communication qui permettra aux membres de faire connaître à la population les diverses problématiques de l'itinérance et les pistes de solution envisagées par le groupe. Faire connaître les activités et les projets du groupe. Favoriser la création d'emplois en permettant aux membres de faire l'apprentissage d'un travail rémunéré dans un milieu leur offrant soutien et encadrement.

Activités: formation liée à l'emploi. Réalisation des activités concernant la rédaction, la production, la promotion et la distribution du journal. Des formations de base sont offertes aux membres qui veulent participer à la rédaction du journal. Des équipes de vendeurs, formées de personnes itinérantes, sont recrutées et encadrées pour assurer la vente du journal dans la rue.

Fréquence des activités: une réunion hebdomadaire du comité de coordination. Les membres participent à un processus d'apprentissage continu (rédaction, informatique, mise en page, etc.). Ils assurent aussi au quotidien la production, la promotion et la distribution du journal. Il y a eu cinq parutions depuis 1992 et, d'ici juin 1995, six autres sont prévues.

Nombre de participants: depuis 1992, environ 100 personnes.

\subsection{Impact et rayonnement du groupe d'entraide}

L'action du groupe l'Itinéraire a engendré des retombées au niveau de I'intervention auprès des personnes itinérantes. Les personnes qui œuvrent dans les organismes du milieu de l'itinérance et dans les différentes instances gouvernementales qui ont été en contact avec les membres du groupe ne perçoivent plus les personnes itinérantes seulement comme des personnes qui ont des problèmes et qui sont toujours en attente d'une réponse à leurs besoins. Les personnes itinérantes sont aussi maintenant perçues comme des personnes ayant des compétences, capables d'élaborer des pistes de solution et de mettre sur pied des projets pour répondre à leurs propres besoins. La reconnaissance qu'ont obtenue les personnes itinérantes par les activités du groupe leur donne maintenant un pouvoir de négociation face aux décideurs. 
Cette reconnaissance $s^{\prime}$ exprime également par le soutien financier qu'a reçu le groupe pour mettre sur pied ses activités et ses projets. Depuis sa création, le groupe a reçu des subventions du Centre d'accueil Préfontaine (45 000 \$), de la Ville de Montréal (60000\$), du ministère de l'Éducation (3700\$), du journal La Presse (impression gratuite d'une édition spéciale du journal $L^{\prime}$ Itinéraire) et du ministère de la Santé et des Services sociaux (59000\$). Ces diverses sources de financement montrent bien la crédibilité qu'a acquise le groupe ainsi que l'impact et le rayonnement de ses activités.

\section{CONCLUSION}

Plusieurs apprentissages peuvent être tirés de l'expérience décrite dans les pages précédentes. Tout d'abord, une pratique innovatrice telle que I'approche d'empowerment demande des habiletés particulières. Il faut au départ avoir la capacité de faire une intervention professionnelle dans un lieu de travail peu structuré et non encadré. L'intervenant qui travaille dans l'instabilité d'un groupe ouvert est constamment confronté à l'incertitude de son action dans le groupe et à la perception que peut avoir le groupe de son intervention. Deuxièmement, le partage du pouvoir n'est pas quelque chose qui vient facilement à l'intervenant, sa formation d'expert lui conférant d'emblée l'autorité. La présence de contradictions chez l'intervenant quant au désir de contrôle et à la volonté de déléguer du pouvoir est une constante dans son intervention auprès d'un groupe.

Un autre aspect lié à la formation en travail social est que cette dernière présente souvent l'intervention individuelle, l'intervention de groupe et l'organisation communautaire comme des entités divisées; ces divisions résistent mal à la pratique. Par exemple, nous avons mentionné précédemment à quel point les problèmes d'alcoolisme et de toxicomanie des membres du groupe constituaient une difficulté dans la continuité de la démarche entreprise avec ces personnes. II était donc important d'être capable d'intervenir auprès d'un membre du groupe qui rechute, tant pour la survie du groupe que pour le mieuxêtre de la personne. Dans de telles situations, on peut se demander où se situe la ligne de démarcation entre l'intervention individuelle et l'intervention de groupe. La mise sur pied du groupe l'Itinéraire $s^{\prime}$ inscrit dans une recherche de solutions originales aux besoins des personnes itinérantes jugées jusque-là réfractaires aux programmes de réinsertion offerts par les organismes spécialisés. 
L'expérience du groupe l'Itinéraire suggère que I'approche d'empowerment déjà utilisée auprès d'autres clientèles donne des résultats très encourageants même auprès de personnes jugées réfractaires à des interventions plus traditionnelles. Les résultats obtenus montrent que derrière l'image projetée par les personnes itinérantes se cachent des capacités qui n'attendent qu'à être utilisées. Le groupe I'Itinéraire est la preuve que l'apprentissage du pouvoir est non seulement possible, même chez les groupes les plus démunis, mais qu'il est même essentiel pour assurer leur place et leur dignité dans la société. 